\title{
Modular plastic chip for one-shot human papillomavirus diagnostic analysis
}

\author{
G. Vecchio ${ }^{\mathrm{a}, 1}$, S. Sabella ${ }^{\mathrm{b}, 1}$, L. Tagliaferro $^{\mathrm{c}}$, P. Menegazzi $^{\mathrm{c}}$, M.P. Di Bello $^{\mathrm{a}}$, V. Brunetti $^{\mathrm{a}}$, R. Cingolani ${ }^{\mathrm{b}}$, \\ R. Rinaldi ${ }^{\text {, }}$ P.P. Pompa ${ }^{\mathrm{b}, *}$ \\ a National Nanotechnology Laboratory of CNR-INFM, University of Salento, Scuola Superiore ISUFI, 73100 Lecce, Italy \\ ${ }^{\mathrm{b}}$ Italian Institute of Technology, Center for Bio-Molecular Nanotechnology, 73010 Arnesano (Lecce), Italy \\ ${ }^{\mathrm{c}}$ Virology and Molecular Biology Department, Pignatelli Laboratory, 73100 Lecce, Italy
}

\section{A R T I C L E I N F O}

\section{Article history:}

Received 4 August 2009

Available online 18 September 2009

\section{Keywords:}

PCR

HPV

Biochips

Polymeric materials

\begin{abstract}
A B S T R A C T
In this article, we report the design and development of a plastic modular chip suitable for one-shot human papillomavirus (HPV) diagnostics, namely detection of the viral presence and relative genotyping, by two sequential steps performed directly on the same device. The device is composed of two modular and disposable plastic units that can be assembled or used separately. The first module is represented by a polydimethylsiloxane (PDMS) microreactor that is exploited for real-time polymerase chain reaction (PCR) and, thus, is suitable for detecting the presence of virus. The second unit is a PDMS microwell array that allows virus genotyping by a colorimetric assay, based on DNA hybridization technology developed on plastic, requiring simple inspection by the naked eye. The two modules can be easily coupled to reusable hardware, enabling the heating/cooling processes and the real-time detection of HPV. By coupling real-time assay and colorimetric genotyping on the same chip, the assembled device may provide a low-cost tool for HPV diagnostics, thereby favoring the prediction of cancer risk in patients.
\end{abstract}

(C) 2009 Elsevier Inc. All rights reserved.
Human papillomavirus (HPV) $)^{2}$ infections are widely considered to be an important risk factor for the genesis of cervical cancer [1$4]$, which is the second most common tumor disease in women worldwide [5]. Moreover, such infections are etiologically associated with certain types of carcinomas in the head and neck [6]. To date, more than 100 different genotypes have been identified on the basis of the DNA sequence of the L1, E6, and upstream regulatory regions $[2,6,7]$, and depending on the association of the virus with mucosal infections, high-risk (HR), probable medium-risk (MR), and low-risk (LR) categories have been defined [8]. Because of the strong phylogenetic relationship of HPV genotypes to malignant lesions, the knowledge of HPV types is crucial for a correct prognosis, therapy, and formulation of effective vaccines (e.g., virus-like particle HPV vaccine) $[9,10]$ that so far are sensitive, for example, to only specific genotypes of the virus $[11,12]$. Thereby, several molecular biology techniques, based on the molecular detection of HPV infections, have been developed recently. These usually represent important assays because, unlike the classical histological and immunological meth-

\footnotetext{
* Corresponding author. Fax: +39 0832295708.

E-mail address: piero.pompa@unile.it (P.P. Pompa).

1 These authors contributed equally to this work.

2 Abbreviations used: HPV, human papillomavirus; HR, high-risk; MR, medium-risk; LR, low-risk; PCR, polymerase chain reaction; PDMS, polydimethylsiloxane; BSA bovine serum albumin; SDS, sodium dodecyl sulfate; ssDNA, single-stranded DNA; APTES, 3-aminopropyltriethoxysilane; GTA, glutaraldehyde; SSC, sodium chloridesodium citrate; PMT, photomultiplier tube; TBS-T, Tris-buffered saline with Tween; NBT, nitro blue tetrazolium; FRET, fluorescence resonance energy transfer.
}

ods alone, they may lead to unequivocal diagnostics of the presence of infections. Such techniques comprise mainly polymerase chain reaction (PCR) and hybridization tests. Consensus PCR protocols have been conveniently used for large-scale screening clinical analyses, but they can assess only the presence of infections. Although interesting attempts to develop genotype-specific PCR have been carried out and several PCR primer sets enabling the simultaneous amplification of a broad range of HPV fragments have been developed [13-16], the maximum number of detectable types still remains restricted. For this reason, other molecular tests, such as the hybridization-based methodologies (e.g., restriction fragment length polymorphism [17,18], type-specific probe hybridization [19], reverse hybridization [20-22]) and sequencing [23-25], must be applied to the amplified solutions to achieve the unambiguous identification of the highest number of genotypes. Importantly, because all of such tests show advantages and disadvantages, a combination of amplification methods and molecular recognition ones and/or sequencing may be desirable for in-depth diagnostics. Notably, due to their high parallelism of analysis, miniaturized biochips suitable for HPV analysis (e.g., microarray-based lab-on-a-chip) may offer an appealing chance to perform highly sensitive diagnostic tests. Moreover, the intrinsic advantages provided by biochips (e.g., portability, facile handling, savings of biological samples, point-of-care applicability) may permit their diffusion as diagnostic devices for large-scale screening programs not only in centralized laboratories but also in peripheral centers such as medical offices and public centers. For example, DNA microarray biochips have 
demonstrated the detection of single infections and even multiple infections simultaneously with high sensitivity [26-30]. Such devices have been developed on different materials (e.g., nylon membrane, glass), and a discrete number of genotypes have been characterized by a simple colorimetric detection of the hybridization between type-specific probes and target.

In this article, we describe the development of a biochip with the ability to conjugate more molecular HPV tests (e.g., real-time PCR, reverse hybridization) on a small-sized device, showing the unique advantage to providing more diagnostic information at once. The device has two plastic modular units, namely a polydimethylsiloxane (PDMS) microreactor (for real-time PCR) and a PDMS microwell array that allows virus genotyping (if the infection has been detected in the first module) by a simple colorimetric assay. Being disposable, the modular units can be used in association or separately on supporting hardware, providing a novel inexpensive tool for HPV diagnostics, likely favoring the prediction of cancer risk in patients.

\section{Materials and methods}

\section{PDMS microreactors}

The PDMS microreactors were produced by means of a simple soft lithography technique, based on replica molding, as described by Sabella and coworkers [31]. To produce microreactors entirely of PDMS, the method was modified slightly as described briefly below. A solution consisting of PDMS (Sylgard 184, Dow Corning, Midland, MI, USA) and curing agent in a 10:1 (w/w) ratio was thoroughly mixed, sonicated for degassing, and poured into forming molds and punchers, conferring the desired shape and dimensions to microreactors. After curing in an oven at $70^{\circ} \mathrm{C}$ for $2 \mathrm{~h}$, the PDMS microreactors were peeled off. Very thin PDMS layers, acting as the bottom of the microreactors, were then produced by spin-coating. The thickness of the bottom layer was investigated extensively and optimized (it was in the range of a few hundred micrometers) to guarantee a proper thermal cycling of the PCR solution. Afterward, PDMS microreactors were made hydrophilic by means of oxygen plasma (PICO, Diener Electronic, Nagold, Germany; experimental conditions: $25 \mathrm{~W}$ for $2 \mathrm{~min}$ ) and immediately blocked with bovine serum albumin (BSA) and sodium dodecyl sulfate (SDS), both at a concentration of $0.1 \%$. Microreactors were then washed extensively with Milli-Q water. The BSA-based treatment was found to be extremely effective and stable (the microreactors typically preserved their stability and biocompatibility over several months). Interestingly, after passivation, PDMS microreactors can be sterilized by conventional autoclaves without losing any surface feature and biocompatibility. The efficiency of thermal cycling was evaluated experimentally by using different thermocouples inside the PCR solution in the plastic microreactors. Specifically, the experiments reported in this article (including real-time PCR and PCR with end-point detection by gel electrophoresis) were performed on square- and cylinder-shaped single-well microreactors with a volume capacity of $30 \mu \mathrm{l}$.

\section{PDMS microwell array for HPV genotyping}

The PDMS microwell array consists of two assembled PDMS slides. To obtain a controlled thickness $(\sim 500 \mu \mathrm{m})$ of the slides, the PDMS/curing agent mixture (1:10 ratio) was spin-coated (Laurell WS $650 \mathrm{~S}-6 \mathrm{NPP} /$ Lite, $300 \mathrm{rpm} \times 20 \mathrm{~s}$ ) on rigid flat masters. After curing at $70^{\circ} \mathrm{C}$ for $2 \mathrm{~h}$, the polymeric films were peeled off. A number of wells (typically 6-35 wells with a capacity ranging from 2 to $5 \mu \mathrm{l}$ ) were produced on the first slide by micropunching the sur- face, whereas the second slide, acting as the bottom, was assembled with the first one by oxygen plasma [32].

The polymeric wells were chemically modified to permit the covalent immobilization of different types of single-stranded DNA (ssDNA) probe molecules according to a published procedure [33] that was slightly modified by us. Briefly, after oxygen plasma treatment, the microwell array was immediately immersed in a $10 \%(\mathrm{v} / \mathrm{v})$ aqueous solution of 3-aminopropyltriethoxysilane (APTES, Sigma-Aldrich, St. Louis, MO, USA) at $25^{\circ} \mathrm{C}$ for $3 \mathrm{~h}$, rinsed with water, and finally dried with a stream of $\mathrm{N}_{2}$. APTES reacts with the hydroxyl groups on PDMS to yield primary amino functional groups on the surface. The aminosilanized PDMS surface (PDMS$\mathrm{NH}_{2}$ ) was activated with the homo-bifunctional linker glutaraldehyde (GTA, Sigma-Aldrich), suitable to react with aminated probes such as $5^{\prime}$-aminated ssDNA. We used solutions of GTA at a concentration of $2.5 \%(\mathrm{v} / \mathrm{v})$ in $100 \mathrm{mM}$ phosphate buffer, and reactions were carried out at $4{ }^{\circ} \mathrm{C}$ in the dark for $2 \mathrm{~h}$. Afterward, substrates were washed abundantly with Milli-Q water and dried with a stream of $\mathrm{N}_{2}$.

We immobilized in the microwells two classes of molecules: probes that were type specific for four types of HPV (HPV probes) and molecule used as a standard control (HPV test). Specifically, the HPV probes were amino-modified at the $5^{\prime}$ end. Such probes had sequences that were specific for HPV 6, 16, 31, and 51, henceforth referred to as HPV-6 probe (5'-TAT TTG TTG GGG TAA TCA ACT G-3'), HPV-16 probe (5'-CAT TTG TTG GGG TAA CCA ACT A$\left.3^{\prime}\right)$, HPV-31 probe (5'-TAT TTG TTG GGG CAA TCA GTT A-3'), and HPV-51 probe (5'-CAT TTG CTG GAA CAA TCA GCT T-3'), respectively. The HPV test was indeed amino-modified at the $5^{\prime}$ end, and biotin was modified at the $3^{\prime}$ end. Such sequence, referred to as HPV-test (5'-CGG AAG CAG GCC CAA TCT GCA A-3'), was used as a standard control for the colorimetric assay because it selectively binds the streptavidinated enzyme. All probes were purchased from Thermo Fisher. ssDNA molecules $(30 \mu \mathrm{M}$ in $3 \times$ SSC [sodium chloride-sodium citrate] buffer) were immobilized on the bottom of each well by soaking solutions at $25^{\circ} \mathrm{C}$ for $30 \mathrm{~min}$, followed by another incubation at $70{ }^{\circ} \mathrm{C}$ for $60 \mathrm{~min}$. Afterward, wells were rinsed thoroughly with $0.2 \%$ SDS solution at $25^{\circ} \mathrm{C}$ for 2 min, washed with Milli-Q water for 2 min, and then blocked with a solution of $\mathrm{NaBH}_{4}$ at $37^{\circ} \mathrm{C}$ for $1 \mathrm{~h}$, followed by extensive washing with a solution of $0.2 \%$ SDS and water.

The optimal surface density of the immobilized ssDNA within the wells was assessed and quantified by fluorescence measurements. Standard solutions of Cy3-modified ssDNA HPV probes ranging from 0.001 to $50 \mu \mathrm{M}$ were spotted on GTA-functionalized PDMS slides. By confocal microscopy analyses (Leica, TCS-SP5 AOBS), we found out that the highest fluorescence signal was obtained with the $30-\mu \mathrm{M}$ value (data not shown).

\section{Assembling of the HPV prototype device}

The overall HPV diagnostics was carried out on a homemade assembled system. The plastic microreactors and microwell array were fixed on the system by simply contacting them with the surface of a thermal plate for PCR thermal cycling (flat plate, Techne). Fluorescence detection of real-time PCR was performed with a low-power excitation source (e.g., a blue LED) and a bandpass filter and photomultiplier tube (PMT) detector in a classical right-angle geometry. Microreactors were used as the disposable part of the diagnostic system so that they were changed for each analysis.

\section{Validation tests of PDMS microreactors and HPV real-time PCR}

Prior to performing the amplification experiments with HPV DNA sequences extracted from biological samples, the plastic microreactors were validated extensively in terms of biocompatibility 
and efficiency by carrying out several PCRs on human genomic DNA with end-point gel electrophoresis detection. In particular, we used as a model target a 206-bp sequence of the CDK11 gene from human genomic DNA (length $\sim 10^{9} \mathrm{bp}$ ) that was amplified by the following PCR master mix: approximately $45 \mu \mathrm{l}$ or less of MegaMix (Microzone), $0.4 \mu \mathrm{M}$ of primer mix (forward: $5^{\prime}$-AGTG CTTCCCTTGCTTTCAA-3'; reverse: 5'-TGTGCTGTACATTTCCTGTGT $\left.\mathrm{G}-3^{\prime}\right)$, and $1.5 \mathrm{ng} / \mu \mathrm{l}$ of DNA template. All reagents were purchased from Sigma-Aldrich. PCR experiments with end-point detection (250 independent analyses) were performed simultaneously in vials (used as reference) and in microreactors inside a thermal cycler ( $\mathrm{T}$ 3000, Biometra, Goettingen, Germany) using aliquots of the same master mix. Solutions were analyzed by agarose gel electrophoresis by using O'GeneRuler 1 kb DNA (Fermentas Life Sciences) as marker. Validation was carried out by analyzing two analytical parameters, namely specificity and efficiency (band position and PCR yield, respectively). Quantification of DNA, migrated as a band in the gel, was calculated by means of one-dimensional gel analysis software (UVIpro Platinum 1.1).

Real-time PCR measurements were performed on real biological samples, that is, DNA extracted from patients affected by various types of $\operatorname{HPV}(6,16,31$, and 51$)$ kindly provided by the Pignatelli Laboratory (Lecce, Italy). Such samples were previously characterized by commercial instrumentation and tested in our plastic device without knowing the virus type a priori. Results were then compared with standard protocols only at the end of the colorimetric response of our plastic multiwell array. In real-time PCR experiments, a gene fragment containing a sequence of approximately $65 \mathrm{bp}$ was amplified. We used the same master mix of the PCR with end-point detection (see above) except for the four forward primers (HP 1E, HP 1F, HP 1G, and HP 1H) and three biotinylated reverse primers (HP 2Cbiot, HP 2Dbiot, and HP 2Ebiot) [34], enabling the amplification of fragments related to the L1 region of $39 \mathrm{HPV}$ genotypes. The fluorescent dye SYBR Green I $\left(\lambda_{\mathrm{ex}}=488 \mathrm{~nm}, \lambda_{\mathrm{em}}=522 \mathrm{~nm}\right)$ was added to the master mix. For real-time PCR analyses, we employed a square-shaped PDMS microreactor. We typically collected the fluorescence signal of SYBR Green during the annealing phase of each PCR cycle by using $0.5 \mathrm{~s}$ excitation and simultaneous detection by a PMT. To assess the yield and specificity of PCR amplification, we carried out additional end-point detection (after 40 cycles) by means of agarose gel electrophoresis. The marker used to analyze amplicon solutions was the PCR 20-bp Low Ladder (Sigma-Aldrich), presenting a size range of $20-1000 \mathrm{bp}$.

\section{HPV genotyping by PDMS microwell array}

In the case of positive detection of HPV, the final PCR products containing the biotinylated amplimers (obtained during the PCR reaction [see above]) were characterized by hybridization with the HPV type-specific probes immobilized in the wells of the PDMS array. The array was soaked with the PCR solution, which was diluted 10 -fold with a buffer solution ( $9 \times$ SSC and $0.1 \%$ SDS), denatured at $95{ }^{\circ} \mathrm{C}$ for $5 \mathrm{~min}$, and finally maintained at $42{ }^{\circ} \mathrm{C}$ for $30 \mathrm{~min}$ to allow hybridization with the solid-state HPV probes. After incubation, the solution was gently removed and the PDMS array was washed thoroughly by three washing cycles with solutions composed of $2 \times$ SSC $0.2 \%$ SDS, $2 \times$ SSC, and $0.2 \times$ SSC, respectively, at $25^{\circ} \mathrm{C}$ for $10 \mathrm{~min}$. Afterward, the microwell array was rinsed immediately with streptavidin-alkaline phosphatase enzyme solution in $0.05 \%$ TBS-T (Tris-buffered saline with Tween, Sigma-Aldrich) at a concentration ratio of 1:1000 for $10 \mathrm{~min}$. After washing the substrates with the same buffer for $2 \mathrm{~min}$, wells were incubated for $30 \mathrm{~min}$ at $25^{\circ} \mathrm{C}$ with the BCIP/NBT (nitro blue tetrazolium) Liquid Substrate System (Sigma-Aldrich). Finally, the BCIP/ NBT was removed and the array was dried in an oven. The reaction mechanism of such colorimetric substrate is based on the production of an insoluble product (NBT diformazan), which deposits as a colored spot in proximity of dsDNA spots on interaction of the enzyme with dsDNA by a biotin-streptavidin bond. Also in this case, to assess the best concentration values, we carried out several experiments by immobilizing in the wells solutions of $5^{\prime}$ aminoand $3^{\prime}$ biotin-modified ssDNA probes, following the same procedures described for the HPV probes. We tested enzyme solutions ranging from a concentration ratio of 1:50 up to 1:5000 with different incubation times $(10,20$, and $30 \mathrm{~min})$, finding out that the best value corresponded to a $1: 1000$ concentration with $10 \mathrm{~min}$ of incubation time.

\section{Results and discussion}

In this article, we show the realization of a plastic, disposable, and modular chip suitable for one-shot HPV diagnostics, namely detection of the viral infection and relative genotyping, by two sequential steps performed directly on the same device. Fig. 1 shows the modular chip we realized, which is composed of two disposable plastic units (i.e., a microreactor and a microwell array) combined with reusable hardware consisting of a heating/cooling surface and a simple optical apparatus for fluorescence detection. Plastic transparent microreactors and PDMS microwell arrays can be produced easily and cheaply by means of soft lithography procedures such as replica molding and micropunching (see Materials and methods for details). Depending on sizes and shapes, the disposable plastic reactors can be easily adapted and used for amplification reactions or as colorimetric plastic microwell arrays (Fig. 1A). By setting up the fabrication parameters as well as the surface treatments (physical and chemical) to obtain hydrophilic and biocompatible surface properties, we produced plastic units presenting very good versatility and applicability for genomic analysis. Such plastic modules are ideal for real-time PCR and virus recognition and genotyping. In particular, for the experiments presented here, we used square- and cylinder-shaped single-chamber PDMS microreactors with a volume capacity of $30 \mu \mathrm{l}$ and PDMS multiwell arrays with $2.5 \mu$ l of working solution (6-35 wells), as reported in Fig. 1B. Microreactors were stably modified and made biocompatible toward PCR by passivating surfaces with oxygen plasma treatments [35] and protein blocking procedures [36] (see Materials and methods for details). On the other hand, microwell arrays were chemically functionalized with HPV probes by means of a surface chemistry modification exploiting APTES reactivity on PDMS and the use of GTA as a linker between the silanized surface and the amino-modified probes (see Materials and methods for details).

Before testing HPV microreactors for real-time PCR of HPV samples, we investigated their performance by an extensive analytical validation, carrying out PCR on human genomic DNA (250 independent experiments) with end-point detection. Notably, we obtained very good results in terms of specificity and efficiency of the microreactors as compared with standard vials in thermal cyclers (Fig. 2). Experimental data show that microreactors well permit the selective amplification of the expected DNA band while avoiding the formation of any aspecific fragments (Fig. 2A). PCR yields are comparable in the two cases (Fig. 2B). Also, these microreactors, which are nearly transparent in the whole visible spectral range, can be used with a wide variety of fluorescent reporters (e.g., molecular beacons, fluorescence resonance energy transfer [FRET] probes) to monitor the real-time progress of the amplification reaction of nucleic acid (real-time PCR). Also, there are virtually no specific constraints on the implementation of the optical setup, with several possible geometries of the excitation/detection scheme. In this work, for HPV detection, we used a widely 

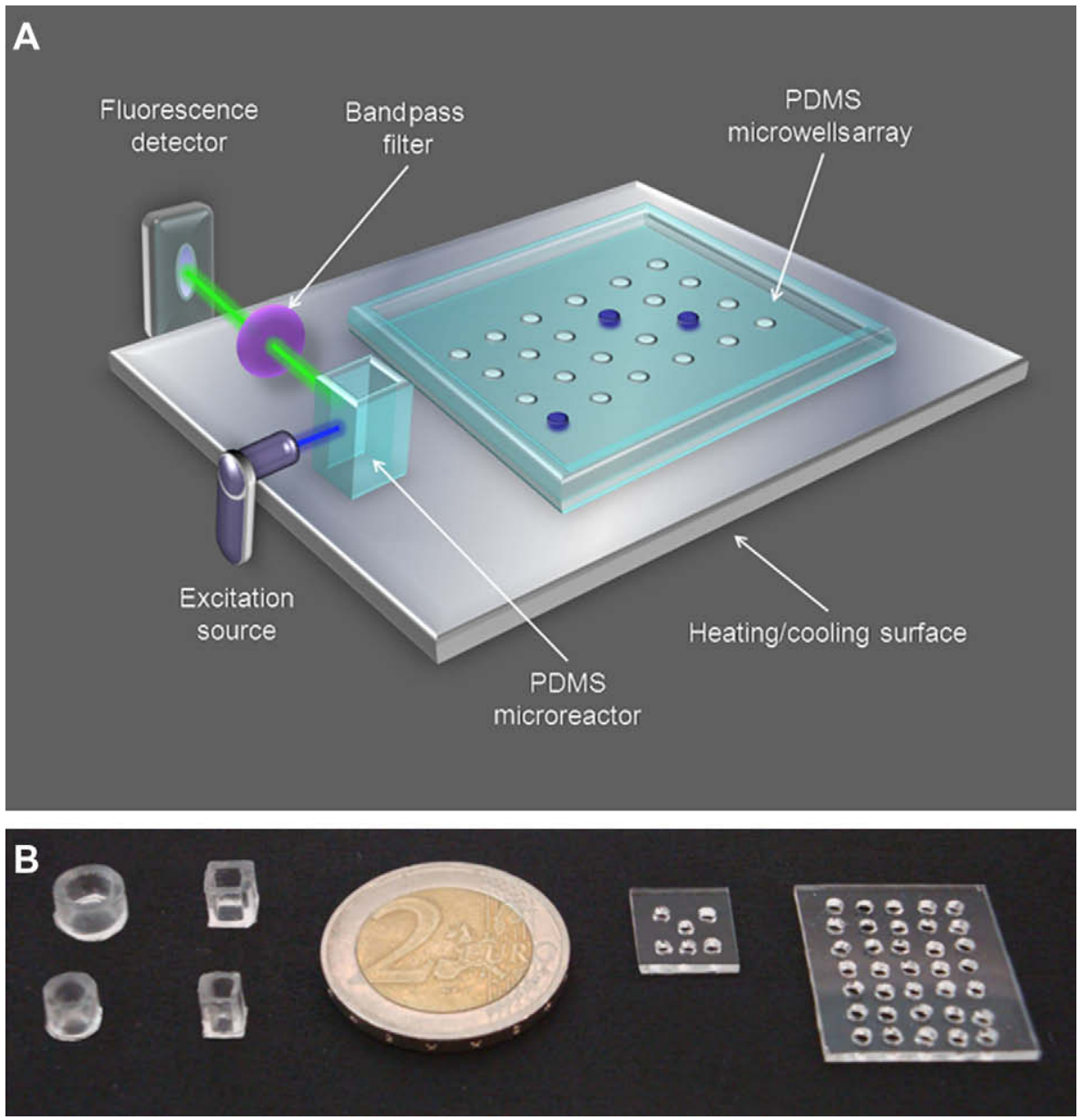

Fig. 1. Modular plastic microreactors and microwell array for diagnostic analyses of HPV. (A) Scheme of the modular HPV prototype device. A square-shaped single-chamber PDMS microreactor and a PDMS multiwell array are positioned on a heating/cooling surface, enabling specific thermal ramps. The transparent disposable microreactor is illuminated by a low-power light source (e.g., an LED), and the fluorescence detector in a right-angle geometry collects the emitted fluorescence for real-time measurement during PCR analysis. (B) Representative photograph of square- and cylinder-shaped single-chamber microreactors with different volume capacities (up $50 \mu \mathrm{l}$, bottom $30 \mu \mathrm{l}$ ) and multiwell arrays with the number of wells ranging from 6 to 35 (maximum volume capacity $5 \mu \mathrm{l}$ ). The inner walls of each well are covalently functionalized with HPV type-specific DNA probes.
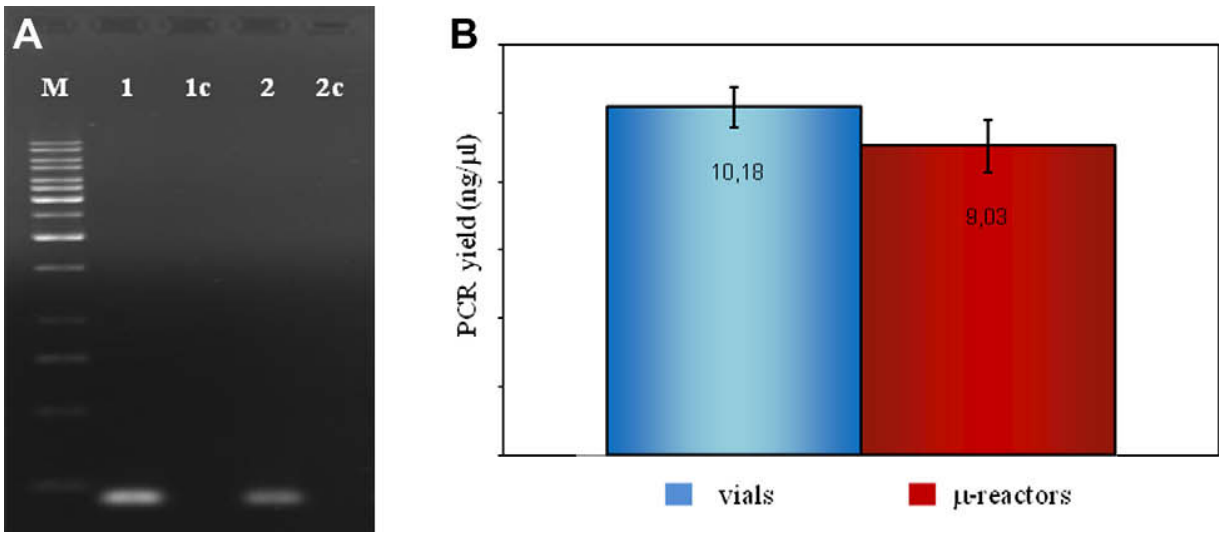

Fig. 2. Analytical validation of microreactors for PCR of human genomic DNA (206-bp fragment of CDK11 gene). A total of 250 independent experiments were performed in standard vials (control) and in microreactors in thermal cyclers. (A) Representative image of the performed gels. Lane M: marker (O'GeneRuler $1 \mathrm{~kb}$ DNA); lane 1: vials; lane 1c: negative control in vials; lane 2: microreactors; lane 2c: negative control in microreactors. As it is possible to observe from the selected gel, the amplified DNA fragments show the expected molecular mass size ( 206 bp) and no detectable aspecific bands, thereby indicating very good specificity of the reaction (such high PCR specificity was observed in all 250 experiments). (B) Histograms reveal that the microreactors show an efficiency comparable to the reference commercial instrumentation. Histogram values represent averages from 250 independent experiments, and error bars report the standard deviations.

exploited fluorescent reporter, namely SYBR Green I, and a classical right-angle geometry for the optical scheme coupled to a square- shaped PDMS microreactor (Fig. 3A), easily fixed on the thermal plate for cycling PCR thermal ramps. In any case, our PDMS mic- 

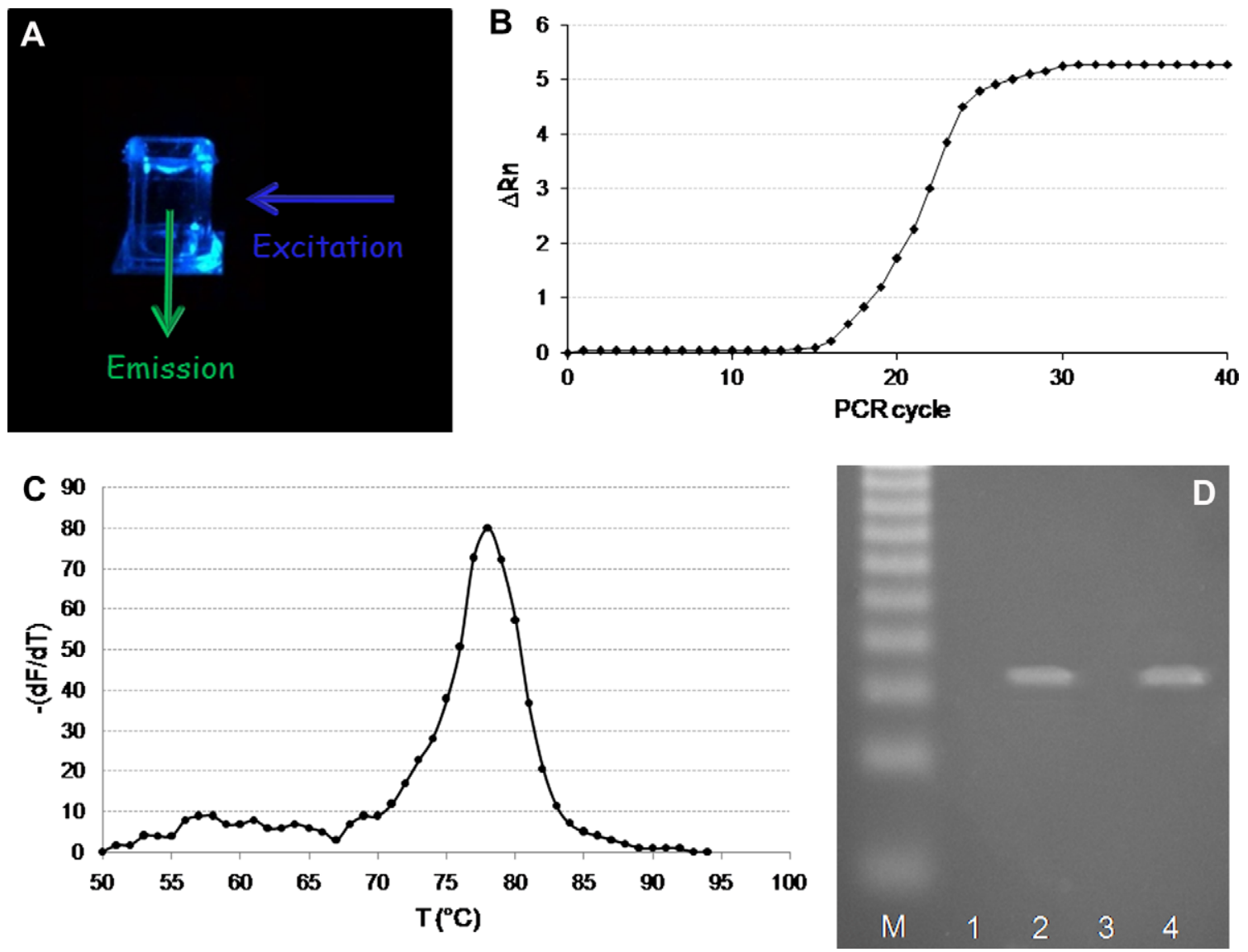

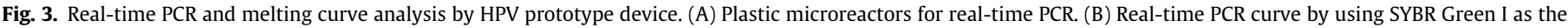

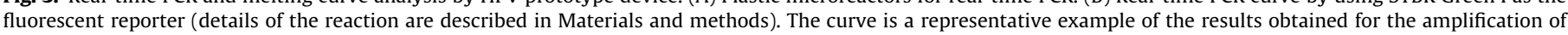

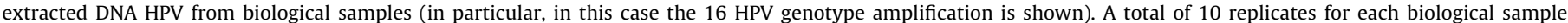

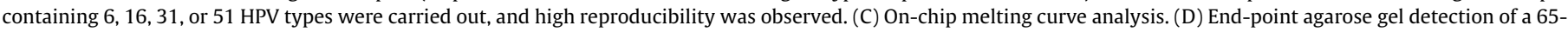

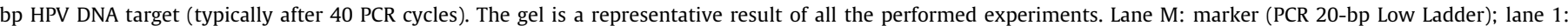

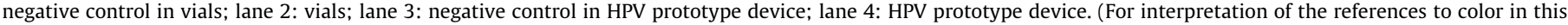
figure legend, the reader is referred to the web version of this paper.)

roreactors are compatible with a wide range of heating/cooling miniaturized systems (e.g., commercially available systems, Peltier or miniaturized microheaters, whose technology is currently well established $[37,38])$ to perform efficient and fast PCR reactions. Real-time PCR was carried out on human DNA extracted from biological samples of patients with HPV infections (Fig. 3). We focused on the L1 HPV region, which was used to amplify a target sequence of approximately 65 bp specific for at least 39 HPV types. In this experiment, we typically collected the fluorescence signal of SYBR Green during the annealing phase of each PCR cycle. Due to the primer specificity for HPV fragments, the typical sigmoid-like shape of the real-time PCR curve revealed the presence of the infection, as shown in Fig. 3B. Importantly, our plastic device allows melting curve analysis, providing the interesting opportunity to assess the specificity of the amplification in situ, as shown in Fig. 3C. In this case, we collected the SYBR Green fluorescence signal in the temperature range from 50 to $94^{\circ} \mathrm{C}$, observing a melting temperature of approximately $78^{\circ} \mathrm{C}$, in line with the expected melting temperature for HPV amplicons [34]. Importantly, real-time PCR data were consistent with gel electrophoresis analysis performed at the end of the reaction (typically after 40 PCR cycles [see Fig. 3D]), further confirming the DNA band specificity and PCR yield.

If virus presence is detected by real-time PCR in the microreactor module, the second plastic unit may be used for genotyping analysis. Hence, the just amplified PCR products were directly ana- lyzed by the PDMS microwell array, which allows colorimetric detection of various HR or LR HPV types. A schematic illustration of the detection principle is reported in Fig. 4A. Reverse DNA hybridization between specific PCR products (which are biotinylated amplimers) and HPV probes (which are selectively immobilized in the PDMS microwell array) is detected by means of an enzymatic reaction producing colored spots. In particular, the reaction occurs between streptavidin-alkaline phosphatase, which selectively binds only the hybridized target DNA by the biotinstreptavidin bond, and the specific substrate, namely the BCIP/ NBT Liquid Substrate System. This leads to the formation of localized colored spots on the array, visible by the naked eye, in less than 10 min due to the deposition of NBT diformazan precipitate [39]. Therefore, by analyzing the pattern of the colored spot on the basis of the immobilized DNA probe sequences, specific for some HR, MR, and LR HPV types, it is possible to genotype the DNA target. As proof of principle, we immobilized HR types 16, 31 , and 51 and LR type 6 . To optimize the colored spot signals, we carried out an extensive optimization in terms of optimal probe density, enzyme concentration, and time of reaction. In Fig. $4 \mathrm{~B}, \mathrm{C}^{+}$ represents the positive control (i.e., biotin-modified DNA probe showing high specificity for the enzyme and no affinity for the DNA target), which was used as a standard control for the enzyme reactivity (see Materials and methods), and $\mathrm{C}^{-}$represents the negative control, which does not contain any immobilized DNA probes. The numbered wells $6,16,31$, and 51 correspond to HPV 
A

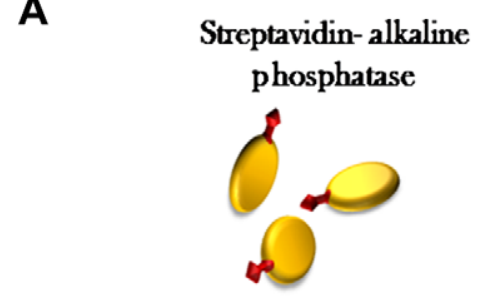

Enzyme substrate

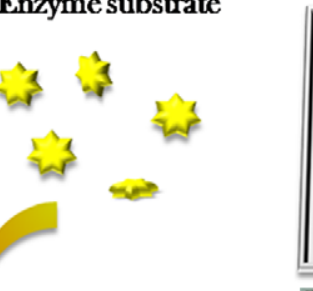

B

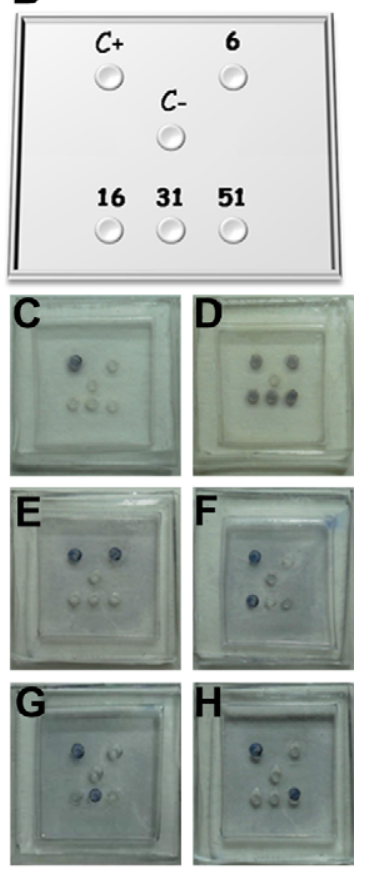

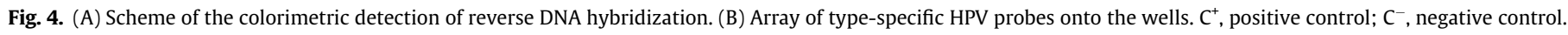

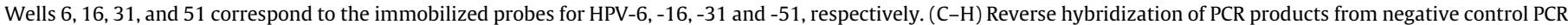

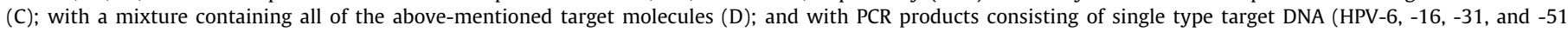
corresponding to panels E-H, respectively).

type-specific probes (Fig. 4B). It can be observed that because $\mathrm{C}^{+}$ selectively reacts with the enzyme, it appears colored in all of the experiments (Fig. 4C-H). In particular, when colorimetric reaction occurs in the absence of target sequences (as reported in Fig $4 \mathrm{C}$, where the chip is tested with PCR products from negative control PCR), only $\mathrm{C}^{+}$spots appear colored, whereas the other wells do not contain any color precipitate, confirming the absence of spurious signals. To demonstrate the ability of our chip to detect multiple infections in a single shot, we produced a mixture solution containing all of the aforementioned HPV target types. As reported in Fig. 4D, all of the wells were colored efficiently except for $\mathrm{C}^{-}$because it did not contain any kind of immobilized probe molecules. Also, single colored spots were evidenced when specific PCR solutions, namely those containing amplicons of each virus type, were allowed to hybridize in the multiwell array chip (Fig. 4E-H report the presence of $6,16,31$, and 51 types, respectively). Therefore, the plastic multiwell array may lead to the precise identification of single HPV types as well as discrimination of multiple targets without any spurious signals. Although the chip was demonstrated to enable identification of four HPV species here, it can be potentially applied to a highly multiplexed colorimetric analysis because no specific fabrication constraints limit the number of wells on the same array.

\section{Conclusions}

We have realized a modular and disposable plastic chip that can be combined with fixed hardware to enable the thermal cycling and real-time detection of HPV amplification. Plastic modules can be used separately or in association, leading to reliable HPV diagnostics for large-scale screening experiments. Both of the plastic modules, the microreactor and the multiwell array, can be produced inexpensively by in-batch fabrication. They are disposable, transparent, and easily adaptable to miniaturized hardware. Their wide applicability for genomic analysis (e.g., detection of viruses and pathogens), as well as for multiplexing colorimetric assays, opens up new perspectives for the use of disposable and miniaturized plastic chips in point-of-care analysis.

\section{Acknowledgments}

The authors gratefully acknowledge S. Shiv Shankar for useful discussion and E. Perrone and V. Fiorelli for expert technical assistance. This work was supported by the Italian Ministry of Research through the MIUR “FIRB” project (RBLA03ER38_001).

\section{References}

[1] H. zur Hausen, Condylomata acuminata and human genital cancer, Cancer Res. 36 (1976) 794.

[2] H. zur Hausen, Papillomavirus infections: a major cause of human cancers, Biochim. Biophys. Acta 1288 (1996) F55-F78.

[3] H.C. Lai, C.A Sun, M.H. Yu, H.J. Chen, H.S. Liu, T.Y. Chu, Favorable clinical outcome of cervical cancers infected with human papilloma virus type 58 and related types, Int. J. Cancer 84 (1999) 553-557.

[4] H.J. Huang, S.L. Huang, C.Y. Lin, R.W. Lin, F.Y. Chao, M.Y. Chen, T.C. Chang, S. Hsueh, K.H. Hsu, C.H. Lai, Human papillomavirus genotyping by a polymerase chain reaction-based genechip method in cervical carcinoma treated with neoadjuvant chemotherapy plus radical surgery, Int. J. Gynecol. Cancer 14 (2004) 639-649.

[5] D.M. Parkin, F.I. Bray, S.S. Devesa, Cancer burden in the year 2000: the global picture, Eur. J. Cancer 37 (2001) S4-S66.

[6] M. Frisch, R.J. Biggar, Aetiological parallel between tonsillar and anogenital squamous-cell carcinomas, Lancet 354 (1999) 1442-1443.

[7] S.Y. Chan, H. Delius, A.L. Halpern, H.U. Bernard, Analysis of genomic sequences of 95 papillomavirus types: uniting typing, phylogeny, and taxonomy, J. Virol. 69 (1995) 3074-3083.

[8] N. Munoz, F.X. Bosch, S. de Sanjose, R. Herrero, X. Castellsague, K.V. Shah, P.J. Snijders, C.J. Meijer, Epidemiologic classification of human papillomavirus types associated with cervical cancer, N. Engl. J. Med. 348 (2003) 518-527.

[9] L.L. Villa, L. Sichero, P. Rahal, O. Caballero, A. Ferenczy, T. Rohan, E.L. Franco, Molecular variants of human papillomavirus types 16 and 18 preferentially associated with cervical neoplasia, J. Gen. Virol. 81 (2000) 2959-2968. 
[10] D.M. Cerqueira, T. Raiol, N.M. Veras, N. von Gal Milanezi, F.A. Amaral, M. de Macedo Brigido, C.R. Martins, New variants of human papillomavirus type 18 identified in central Brazil, Virus Genes 37 (2008) 282-287.

[11] A. Ames, P. Gravitt, Human papillomavirus vaccine update, Curr. Infect. Dis. Rep. 9 (2007) 151-158.

[12] J.T. Schiller, X. Castellsague, L.L. Villa, A. Hildesheim, An update of prophylactic human papillomavirus L1 virus-like particle vaccine clinical trial results, Vaccine 26 (2008) K53-K61.

[13] W. Qu, G. Jiang, Y. Cruz, C.J. Chang, G.Y. Ho, R.S. Klein, R.D. Burk, PCR detection of human papillomavirus: Comparison between MY09/MY11 and GP5+/GP6+ primer systems, J. Clin. Microbiol. 35 (1997) 1304-1310.

[14] P.E. Gravitt, C.L. Peyton, T.Q. Alessi, C.M. Wheeler, F. Coutlee, A. Hildesheim, M.H. Schiffman, D.R. Scott, R.J. Apple, Improved amplification of genital human papillomaviruses, J. Clin. Microbiol. 38 (2000) 357-361.

[15] B. Kleter, L.J. van Doorn, J. ter Schegget, L. Schrauwen, K. van Krimpen, M Burger, B. ter Harmsel, W. Quint, Novel short-fragment PCR assay for highly sensitive broad-spectrum detection of anogenital human papillomaviruses, Am. J. Pathol. 153 (1998) 1731-1739.

[16] M.V. Jacobs, P.J. Snijders, A.J. van den Brule, T.J. Helmerhorst, C.J. Meijer, J.M. Walboomers, A general primer GP5+/GP6(+)-mediated PCR-enzyme immunoassay method for rapid detection of 14 high-risk and 6 low-risk human papillomavirus genotypes in cervical scrapings, J. Clin. Microbiol. 35 (1997) 791-795.

[17] T. Meyer, R. Arndt, E. Stockfleth, H.T. Flammann, H. Wolf, U. Reischl, Strategy for typing human papillomaviruses by RFLP analysis of PCR products and subsequent hybridization with a generic probe, BioTechniques 19 (1995) 632639.

[18] M. Grce, K. Husnjak, M. Skerlev, J. Lipozencic, K. Pavelic, Detection and typing of human papillomaviruses by means of polymerase chain reaction and fragment length polymorphism in male genital lesions, Anticancer Res. 20 (2000) 2097-2102.

[19] N. Ylitalo, T. Bergstrom, U. Gyllensten, Detection of genital human papillomavirus by single-tube nested PCR and type-specific oligonucleotide hybridization, J. Clin. Microbiol. 33 (1995) 1822-1828.

[20] P.E. Gravitt, C.L. Peyton, R.J. Apple, C.M. Wheeler, Genotyping of 27 human papillomavirus types by using L1 consensus PCR products by a singlehybridization, reverse line blot detection method, J. Clin. Microbiol. 36 (1998) 3020-3027.

[21] B. Kleter, L.J. van Doorn, L. Schrauwen, A. Molijn, S. Sastrowijoto, J. ter Schegget, J. Lindeman, B. ter Harmsel, M. Burger, W. Quint, Development and clinical evaluation of a highly sensitive PCR-reverse hybridization line probe assay for detection and identification of anogenital human papillomavirus, J Clin. Microbiol. 37 (1999) 2508-2517.

[22] A.J. van den Brule, R. Pol, N. Fransen-Daalmeijer, L.M. Schouls, C.J. Meijer, P.J. Snijders, GP5+/6+ PCR followed by reverse line blot analysis enables rapid and high-throughput identification of human papillomavirus genotypes, J. Clin. Microbiol. 40 (2002) 779-787.

[23] S.D. Vernon, E.R. Unger, D. Williams, Comparison of human papillomavirus detection and typing by cycle sequencing, line blotting, and hybrid capture, J. Clin. Microbiol. 38 (2000) 651-655.

[24] S. Kosel, S. Burggraf, J. Mommsen, W. Engelhardt, B. Olgemoller, Type-specific detection of human papillomaviruses in a routine laboratory setting: improved sensitivity and specificity of PCR and sequence analysis compared to direct hybridisation, Clin. Chem. Lab. Med. 41 (2003) 787-791.
[25] N. Speich, C. Schmitt, R. Bollmann, M. Bollmann, Human papillomavirus (HPV) study of 2916 cytological samples by PCR and DNA sequencing: genotype spectrum of patients from the West German area, J. Med. Microbiol. 53 (2004) 125-128.

[26] N.H. Cho, H.J. An, J.K. Jeong, S. Kang, J.W. Kim, Y.T. Kim, T.K. Park, Genotyping of 22 human papillomavirus types by DNA chip in Korean women: comparison with cytologic diagnosis, Am. J. Obstet. Gynecol. 188 (2003) 56-62.

[27] T.J. Oh, C.J. Kim, S.K. Woo, T.S. Kim, D.J. Jeong, M.S. Kim, S. Lee, H.S. Cho, S. An, Development and clinical evaluation of a highly sensitive DNA microarray for detection and genotyping of human papillomaviruses, J. Clin. Microbiol. 42 (2004) 3272-3280.

[28] Y.D. Choi, W.W. Jung, J.H. Nam, H.S. Choi, C.S. Park, Detection of HPV genotypes in cervical lesions by the HPV DNA chip and sequencing, Gynecol. Oncol. 98 (2005) 369-375.

[29] H. Lin, J.S. Moh, Y.C. Ou, S.Y. Shen, Y.M. Tsai, C.C. ChangChien, J.M. Liu, Y.Y. Ma, A simple method for the detection and genotyping of high-risk human papillomavirus using seminested polymerase chain reaction and reverse hybridization, Gynecol. Oncol. 96 (2005) 84-91.

[30] C.Y. Lin, H.C. Chen, R.W. Lin, S.L. You, C.M. You, L.C. Chuang M.H. Pan, M.H. Lee Y.C. Chou, C.J. Chen, Quality assurance of genotyping array for detection and typing of human papillomavirus, J. Virol. Methods 140 (2007) 1-9.

[31] S. Sabella, G. Vecchio, R. Cingolani, R. Rinaldi, P.P. Pompa, Real-time PCR in a plastic chip based on solid state FRET, Langmuir 24 (2008) 13266-13269.

[32] D.C. Duffy, J.C. McDonald, O.J.A. Schueller, G.M. Whitesides, Rapid prototyping of microfluidic systems in poly(dimethylsiloxane), Anal. Chem. 70 (1998) 4974-4984.

[33] A. Fung, C.Y. Yang, S. Freire, C. Montemagno, B. Brough, C.M. Ho, F. Gu, W. Shi, Fluorescent detection of oral pathogens by a solid-phase immunoassay on PDMS, Conf. Proc. IEEE Eng. Med. Biol. Soc. 3 (2005) 2630-2633.

[34] C. Payan, A. Ducancelle, M.H. Aboubaker, J. Caer, M. Tapia, A. Chauvin, D. Peyronnet, E. Le Hen, Z. Arab, M.-C. Legrand, A. Tran, E. Postec, F. Tourmen, M. Avenel, C. Malbois, M.-A. De Brux, P. Descamps, F. Lunel, Human papillomavirus quantification in urine and cervical samples by using the Mx4000 and LightCycler general real-time PCR systems, J. Clin. Microbiol. 45 (2007) 897-901.

[35] S. Bhattacharya, A. Datta, J.M. Berg, S. Gangopadhyay, Studies on surface wettability of poly(dimethyl) siloxane (PDMS) and glass under oxygen-plasma treatment and correlation with bond strength, J. Microelectromech. Syst. 14 (2005) 590-597.

[36] C. Consolandi, M. Severgnini, A. Frosini, G. Caramenti, M. De Fazio, F. Ferrara, A Zocco, A. Fischetti, M. Palmieri, G. De Bellis, Polymerase chain reaction of 2-kb cyanobacterial gene and human anti- $\alpha_{1}$-chymotrypsin gene from genomic DNA on the In-Check single-use microfabricated silicon chip, Anal. Biochem. 353 (2006) 191-197.

[37] C.S. Liao, G.B. Lee, H.S. Liu, T.M. Hsieh, C.H. Luo, Miniature RT-PCR system for diagnosis of RNA-based viruses, Nucleic Acids Res. 33 (2005) e156.

[38] C.S. Liao, G.B. Lee, J.J. Wu, C.C. Chang, T.M. Hsieh, F.C. Huang, C.H. Luo, Micromachined polymerase chain reaction system for multiple DNA amplification of upper respiratory tract infectious diseases, Biosens. Bioelectron. 20 (2005) 1341-1348.

[39] M.S. Blake, K.H. Johnston, G.J. Russell-Jones, E.C. Gotschlich, A rapid sensitive method for detection of alkaline phosphatase-conjugated anti-antibody on Western blots, Anal. Biochem. 136 (1984) 175-179. 